\title{
Conduct Problems Scale
}

National Cancer Institute

\section{Source}

National Cancer Institute. Conduct Problems Scale. NCI Thesaurus. Code C121293.

A rating scale included in the Behavior Assessment System for Children that measures the tendency of the subject to engage in antisocial and rule breaking behavior. 